\title{
Central Limit Theorem for Linear Processes with Infinite Variance
}

\author{
Magda Peligrad $d 1$ and Hailin Sang $^{b}$ \\ ${ }^{a}$ Department of Mathematical Sciences, University of Cincinnati, PO Box \\ 210025, Cincinnati, OH 45221-0025, USA. E-mail address: \\ peligrm@ucmail.uc.edu \\ ${ }^{b}$ National Institute of Statistical Sciences, PO Box 14006, Research Triangle \\ Park, NC 27709, USA. E-mail address: sang@niss.org
}

MSC 2000 subject classification: Primary: 60F05, 60G10, 60G42

Key words and phrases: linear process, central limit theorem, martingale, mixing, infinite variance.

\begin{abstract}
This paper addresses the following classical question: giving a sequence of identically distributed random variables in the domain of attraction of a normal law, does the associated linear process satisfy the central limit theorem? We study the question for several classes of dependent random variables. For independent and identically distributed random variables we show that the central limit theorem for the linear process is equivalent to the fact that the variables are in the domain of attraction of a normal law, answering in this way an open problem in the literature. The study is also motivated by models arising in economic applications where often the innovations have infinite variance, coefficients are not absolutely summable, and the innovations are dependent.
\end{abstract}

\section{Introduction and notations}

Let $\left(\xi_{n}\right)_{n \in \mathbb{Z}}$ be a sequence of identically distributed random variables and let $\left(c_{n i}\right)_{1 \leq i \leq m_{n}}$ be a triangular array of numbers. In this paper we analyze the asymptotic behavior of statistics of the type

$$
S_{n}=\sum_{i=1}^{m_{n}} c_{n i} \xi_{i}
$$

when the variables are centered and satisfy:

$$
H(x)=\mathbb{E}\left(\xi_{0}^{2} I\left(\left|\xi_{0}\right| \leq x\right)\right) \text { is a slowly varying function at } \infty .
$$

\footnotetext{
${ }^{1}$ Supported in part by a Charles Phelps Taft Memorial Fund grant and NSA grant H9823009-1-0005
} 
This tail condition is highly relevant to the central limit theory. For independent, identically distributed, centered variables this condition is equivalent to the fact that the variables are in the domain of attraction of the normal law. This means: there is a sequence of constants $b_{n} \rightarrow \infty$ such that $\sum_{i=1}^{n} \xi_{i} / b_{n}$ is convergent in distribution to a standard normal variable (see for instance Feller, 1966; Ibragimov and Linnik, 1971; Araujo and Giné, 1980). It is an open question to extend the general central limit theorem from equal weights to weighted sums of i.i.d. random variables with infinite variance. Linear combinations of identically distributed random variables are important, since many random evolutions and also statistical procedures such as parametric or nonparametric estimation of regression with fixed design, produce statistics of type (11) (see for instance Chapter 9 in Beran, 1994, for the case of parametric regression, or the paper by Robinson, 1997, where kernel estimators are used for nonparametric regression). One example is the simple parametric regression model $Y_{i}=\beta \alpha_{i}+\xi_{i}$ where $\left(\xi_{i}\right)$ is a sequence of identically distributed random variables with marginal distribution satisfying (2),$\left(\alpha_{i}\right)$ is a sequence of real numbers and $\beta$ is the parameter of interest. The least squares estimator $\hat{\beta}$ of $\beta$, based on a sample of size $n$, satisfies $S_{n}=\hat{\beta}-\beta=\left(\sum_{i=1}^{n} \alpha_{i}^{2}\right)^{-1} \sum_{i=1}^{n} \alpha_{i} \xi_{i}$. So the representation of type (11) holds with $c_{n i}=\alpha_{i} /\left(\sum_{i=1}^{n} \alpha_{i}^{2}\right)$.

We shall also see that the asymptotic behavior of the sums of variables of the form

$$
X_{k}=\sum_{j=-\infty}^{\infty} a_{k+j} \xi_{j}
$$

can be obtained by studying sums of the type (1). We shall refer to such a process as to a linear process with innovations $\left(\xi_{i}\right)_{i \in \mathbb{Z}}$. In 1971 Ibragimov and Linnik extended the central limit theorem from i.i.d. random variables to linear processes defined by (3) for innovations that have finite second moment, under the conditions $\sum_{j=-\infty}^{\infty} a_{k}^{2}<\infty$ and $\operatorname{stdev}\left(\sum_{j=1}^{n} X_{j}\right) \rightarrow \infty$. They showed that

$$
\sum_{j=1}^{n} X_{j} / \operatorname{stdev}\left(\sum_{j=1}^{n} X_{j}\right) \stackrel{D}{\rightarrow} N(0,1) .
$$

This result is striking, since $\operatorname{var}\left(\sum_{j=1}^{n} X_{j}\right)$ can be of order different of $n$; practically it can be any positive sequence going to infinite of an order $o\left(n^{2}\right)$. It was conjectured that a similar result might hold without the assumption of finite second moment. Steps in this direction are papers by Knight (1991), Mikosch et al. (1995) and $\mathrm{Wu}(2003)$ who studied this problem under the additional assumption $\sum_{j=-\infty}^{\infty}\left|a_{k}\right|<\infty$. Our Theorem 2.5 positively answers this conjecture. Under condition (2) we show that $X_{k}$ is well defined, if and only if

$$
\sum_{j \in \mathbb{Z}, a_{j} \neq 0} a_{j}^{2} H\left(\left|a_{j}\right|^{-1}\right)<\infty
$$

and under this condition we show that the central limit theorem for $\sum_{j=1}^{n} X_{j}$ properly normalized is equivalent to condition (2). 
As an example in this class we mention the particular linear process with regularly varying weights with exponent $\alpha$ where $1 / 2<\alpha<1$. This means that the coefficients are of the form $a_{n}=n^{-\alpha} L(n)$, where for $n \geq 1, a_{n}=$ 0 for $n \leq 0$, and $L(n)$ is a slowly varying function at $\infty$. It incorporates the fractionally integrated processes that play an important role in financial econometrics, climatology and so on and they are widely studied. Such processes are defined for $0<d<1 / 2$ by

$$
X_{k}=(1-B)^{-d} \xi_{k}=\sum_{i \geq 0} a_{i} \xi_{k-i} \text { where } a_{i}=\frac{\Gamma(i+d)}{\Gamma(d) \Gamma(i+1)}
$$

and $B$ is the backward shift operator, $B \varepsilon_{k}=\varepsilon_{k-1}$. For this example, by the well known fact that for any real $x, \lim _{n \rightarrow \infty} \Gamma(n+x) / n^{x} \Gamma(n)=1$ we have $\lim _{n \rightarrow \infty} a_{n} / n^{d-1}=1 / \Gamma(d)$. Notice that these processes have long memory because $\sum_{j \geq 1}\left|a_{j}\right|=\infty$. This particular class was recently investigated by Peligrad and Sang (2010), where further reaching properties were pointed out.

Our study is not restricted to the class of independent identically distributed random variables. We also consider larger classes including martingales and mixing processes. The results obtained for the class of martingale innovations are also useful to study more general innovations that can be approximated by martingale differences. The martingale approximation method was recently used by Jara et al (2009) to study the attraction to stable laws with exponent $\alpha, \alpha \in(0,2)$ for additive functionals of a stationary Markov chain.

There is a huge literature on the central limit theorem for linear processes with dependent innovations and finite second moment but we are not aware of any study considering the infinite variance case in its full generality. A step in this direction, under the assumption $\sum_{j=-\infty}^{\infty}\left|a_{k}\right|<\infty$, is the paper by TyranKamińska (2010).

In all the central limit theorems for variables with infinite variance the construction of the normalizer is rather complicated and is based heavily on the function $H(x)$. This is the reason why it is important to replace the normalizer by a selfnormalizer, constructed from the data. We mention in this direction the recent results by Kulik (2006), under the assumption that $\sum_{j=-\infty}^{\infty}\left|a_{k}\right|<\infty$ and by Peligrad and Sang (2010) for regularly varying weights with exponent $\alpha$ where $1 / 2<\alpha<1$. In this paper, as in Mason (2005), we suggest a Raikov type selfnormalizer based on a weighted sum of squares of the innovations.

Our paper is organized in the following way: Section 2 contains the definitions and the results, Section 3 contains the proofs. For convenience, in the Appendix, we give some auxiliary results and we also mention some known facts needed for the proofs.

In the sequel we shall use the following notations: a double indexed sequence with indexes $n$ and $i$ will be denoted by $a_{n i}$ and sometimes $a_{n, i}$; we use the notation $a_{n} \sim b_{n}$ instead of $a_{n} / b_{n} \rightarrow 1 ; a_{n}=o\left(b_{n}\right)$ means that $a_{n} / b_{n} \rightarrow 0 ; I(A)$ denotes the indicator function of $A$; the notation $\Rightarrow$ is used for convergence in distribution and also for convergence in probability to a constant. 
In this paper we shall make two conventions in order to simplify the notations.

Convention 1. By convention, for $x=0,|x| H\left(|x|^{-1}\right)=0$. For instance we can write instead $\sum_{j \in \mathbb{Z}, a_{j} \neq 0} a_{j}^{2} H\left(\left|a_{j}\right|^{-1}\right)<\infty$, simply $\sum_{j \in \mathbb{Z}} a_{j}^{2} H\left(\left|a_{j}\right|^{-1}\right)<\infty$.

Convention 2. The second convention refers to the function $H(x)$ defined in (2). Since the case $\mathbb{E}\left(\xi_{0}^{2}\right)<\infty$ is known, we shall consider the case $\mathbb{E}\left(\xi_{0}^{2}\right)=\infty$. Let $b=\inf \{x \geq 0: H(x)>1\}$ and $H_{b}(x)=H(x \vee(b+1))$. Then clearly $b<\infty$, $H_{b}(x) \geq 1$ and $H_{b}(x)=H(x)$ for $x>b+1$. From now on we shall redenote $H_{b}(x)$ by $H(x)$. Therefore, since our results are asymptotic, without restricting the generality we shall assume that $H(x) \geq 1$ for all $x \geq 0$.

\section{Results}

Our first results treat the general weights and identically distributed martingale differences with infinite second moment. The case of finite second moment was treated in Peligrad and Utev $(1997,2006)$.

We shall establish first a general theorem for martingale differences under a convergence in probability condition (5). This condition will be verified in the next main results for classes of martingale differences and i.i.d. random variables.

Theorem 2.1 Let $\left(\xi_{k}\right)_{k \in \mathbb{Z}}$ be a sequence of identically distributed martingale differences adapted to the filtration $\left(\mathcal{F}_{k}\right)_{k \in \mathbb{Z}}$ that satisfy (2) and let $\left(c_{n k}\right)_{1 \leq k \leq m_{n}}$ be a triangular array of real numbers, such that

$$
\sup _{n} \sum_{k=1}^{m_{n}} c_{n k}^{2} H\left(\left|c_{n k}\right|^{-1}\right)<\infty \text { and } \max _{1 \leq k \leq m_{n}} c_{n k}^{2} H\left(\left|c_{n k}\right|^{-1}\right) \rightarrow 0 \text { as } n \rightarrow \infty \text {. }
$$

Assume

$$
\sum_{k=1}^{m_{n}} c_{n k}^{2} \xi_{k}^{2} \Rightarrow 1 \text { as } n \rightarrow \infty
$$

Then

$$
\sum_{k=1}^{m_{n}} c_{n k} \xi_{k} \Rightarrow N(0,1) \text { as } n \rightarrow \infty
$$
(5).

We shall mention two pairwise mixing type conditions that are sufficient for

Proposition 2.1 Assume that all the conditions of Theorem 2.1 (except for (5)) are satisfied. Assume that one of the following two conditions holds:

$\left(M_{1}\right)$ There is a sequence of positive numbers $\psi_{k} \rightarrow 0$ such that for all $a$ and $b$ positive numbers and all integers $j$,

$$
\operatorname{cov}\left(\xi_{j}^{2} I\left(\left|\xi_{0}\right| \leq a\right), \xi_{j+k}^{2} I\left(\left|\xi_{k}\right| \leq b\right)\right) \leq \psi_{k} \mathbb{E}\left(\xi_{0}^{2} I\left(\left|\xi_{0}\right| \leq b\right)\right) \mathbb{E}\left(\xi_{0}^{2} I\left(\left|\xi_{0}\right| \leq a\right)\right) .
$$


$\left(M_{2}\right)$ There is a sequence of positive numbers $\left(\varphi_{k}\right)$ with $\sum_{k \geq 1} \varphi_{k}<\infty$, such that for all $a$ and $b$ and all integers $j$,

$$
\operatorname{cov}\left(\xi_{j}^{2} I\left(\left|\xi_{0}\right| \leq a\right), \xi_{j+k}^{2} I\left(\left|\xi_{k}\right| \leq b\right)\right) \leq a^{2} \varphi_{k} \mathbb{E}\left(\xi_{0}^{2} I\left(\left|\xi_{0}\right| \leq b\right)\right) .
$$

If either $\left(M_{1}\right)$ or $\left(M_{2}\right)$ holds then (5) is satisfied and therefore the conclusion of Theorem 2.1 holds.

Remark 2.1 According to the above proposition we mention that for independent identically distributed innovations satisfying (2) and coefficients $\left(c_{n i}\right)$ satisfying condition (4) the central limit theorem (6) holds.

For further applications to time series we shall comment on the normalized form of the above results, which is important for the case when condition (44) is not satisfied.

Recall Conventions 1 and 2 and define:

$$
D_{n}=\inf \left\{s \geq 1: \sum_{k=1}^{m_{n}} \frac{c_{n k}^{2}}{s^{2}} H\left(\frac{s}{c_{n k}}\right) \leq 1\right\} .
$$

$D_{n}$ is well defined since by Convention $2, H(x) \geq 1$ for all $x$ and, since $H(x)$ is a slowly varying function, we have $\lim _{x \rightarrow \infty} x^{-2} H(x)=0$. By using this definition along with Theorem 2.1 we obtain the following corollary:

Corollary 2.1 Let $\left(\xi_{k}\right)_{k \in \mathbb{Z}}$ be as in Theorem 2.1 and assume

$$
\max _{1 \leq k \leq m_{n}} \frac{c_{n k}^{2}}{D_{n}^{2}} H\left(\frac{D_{n}}{\left|c_{n k}\right|}\right) \rightarrow 0 \text { as } n \rightarrow \infty
$$

and

$$
\frac{1}{D_{n}^{2}} \sum_{k=1}^{m_{n}} c_{n k}^{2} \xi_{k}^{2} \Rightarrow 1 \text { as } n \rightarrow \infty
$$

Then

$$
\frac{1}{D_{n}} \sum_{k=1}^{m_{n}} c_{n k} \xi_{k} \Rightarrow N(0,1) \text { as } n \rightarrow \infty
$$

Moreover, as in Proposition 2.1, condition (9) is satisfied under either $\left(M_{1}\right)$ or $\left(M_{2}\right)$.

Clearly, by combining (9) with (10) we also have

$$
\frac{1}{\left(\sum_{k=1}^{m_{n}} c_{n k}^{2} \xi_{k}^{2}\right)^{1 / 2}} \sum_{k=1}^{m_{n}} c_{n k} \xi_{k} \Rightarrow N(0,1) \text { as } n \rightarrow \infty \text {. }
$$

For equal weights, when $c_{n, i}=1$ for all $n$ and $1 \leq i \leq n, D_{n}$ becomes the standard normalizer for the central limit theorem for variables in the domain of attraction of a normal law:

$$
D_{n}=\inf \left\{s \geq 1: \frac{n}{s^{2}} H(s) \leq 1\right\} .
$$


Then, it is well known that $D_{n} \rightarrow \infty$ and, by the properties of slowly varying function, condition (8) is satisfied with $m_{n}=n$. For this case we easily obtain the following central limit theorem for martingales with infinite variance:

Corollary 2.2 Let $\left(\xi_{k}\right)_{k \in \mathbb{Z}}$ be a sequence of identically distributed martingale differences adapted to the filtration $\left(\mathcal{F}_{k}\right)_{k \in \mathbb{Z}}$ satisfying (2) and assume

$$
\frac{1}{D_{n}^{2}} \sum_{k=1}^{n} \xi_{k}^{2} \Rightarrow 1 \text { as } n \rightarrow \infty
$$

Let $M_{n}=\sum_{k=1}^{n} \xi_{k}$. Then

$$
\frac{M_{n}}{D_{n}} \Rightarrow N(0,1) \text { as } n \rightarrow \infty \text {. }
$$

Moreover, as in Proposition 2.1, condition (12) is satisfied under either $\left(M_{1}\right)$ or $\left(M_{2}\right)$.

Remark 2.2 This corollary can be used to obtain the CLT for classes of stochastic processes that can be approximated by stationary martingale differences. For instance, assume $\left(\eta_{k}\right)_{k \in \mathbb{Z}}$ is a stationary sequence of centered, integrable random variables, $\mathcal{F}_{i}=\sigma\left(\eta_{j}, j \leq i\right)$ and $V_{n}=\sum_{k=1}^{n} \eta_{k}$. An idea, going back to Gordin (1969), is to decompose $V_{n}$ into a martingale with stationary differences and a telescoping rest called coboundary. More precisely, $\eta_{n}=\xi_{n}+Z_{n-1}-Z_{n}$, where $Z_{n}$ is a stationary integrable sequence and $\xi_{n}$ is a stationary sequence of martingale differences. Volný (1993) gave necessary and sufficient conditions for such an approximation. Under the assumption $\mathbb{E}\left(V_{n} \mid \mathcal{F}_{0}\right)$ is convergent in $\mathbb{L}_{1}$, we have $V_{n}=M_{n}+R_{n}$ where $M_{n}$ is a martingale with stationary differences adapted to $\mathcal{F}_{n}$, and $R_{n}=Z_{0}-Z_{n}$ has the property that $E\left|R_{n}\right|<\infty$. Then clearly, after normalizing by a sequence of constants $B_{n}$ converging to $\infty$, the limiting distribution of $V_{n} / B_{n}$ is equivalent to the limiting distribution of $M_{n} / B_{n}$.

By combining our Corollary 2.1 with the main result in Giné et al. (1997) we formulate next a theorem for i.i.d. sequences.

Theorem 2.2 Let $\left(\xi_{k}\right)_{k \in \mathbb{Z}}$ be a sequence of independent and identically distributed centered random variables. Then the following three statements are equivalent:

(1) $\xi_{0}$ is in the domain of attraction of a normal law (i.e. condition (2) is satisfied).

(2) For any sequence of constants $\left(c_{n k}\right)_{1 \leq k \leq m_{n}}$ satisfying (8) the CLT in (10) holds.

(3) For any sequence of constants $\left(c_{n k}\right)_{1 \leq k \leq m_{n}}$ satisfying (8) the selfnormalized CLT in (11) holds.

The implication $(2) \rightarrow(3)$ also follows by Mason (2005). We shall apply our general results for time series of the form (3) with identically distributed martingale differences innovations. We shall prove first the following proposition: 
Proposition 2.2 Let $\left(\xi_{k}\right)_{k \in \mathbb{Z}}$ be a sequence of identically distributed martingale differences adapted to the filtration $\left(\mathcal{F}_{k}\right)_{k \in \mathbb{Z}}$ that satisfy (2). The linear process $X_{0}=\sum_{j=-\infty}^{\infty} a_{j} \xi_{j}$ is well defined in the almost sure sense under the condition

$$
\sum_{j \in \mathbb{Z}} a_{j}^{2} H\left(\left|a_{j}\right|^{-1}\right)<\infty .
$$

If the innovations are independent and identically distributed random variables satisfying (2) then condition (13) is necessary and sufficient for the existence of $X_{0}$ a.s.

Denote

$$
b_{n j}=a_{j+1}+\cdots+a_{j+n}
$$

and with this notation

$$
S_{n}=\sum_{k=1}^{n} X_{k}=\sum_{j \in \mathbb{Z}} b_{n j} \xi_{j} .
$$

Construct $D_{n}$ by (7) where we replace $c_{n j}$ by $b_{n j}$. Then we have:

Theorem 2.3 Assume that $\left(\xi_{k}\right)_{k \in \mathbb{Z}}$ is a sequence of identically distributed martingale differences satisfying (2), the coefficients satisfy condition (13), and $\sum_{k} b_{n k}^{2} \rightarrow \infty$. Assume in addition that

$$
\frac{1}{D_{n}^{2}} \sum_{k} b_{n k}^{2} \xi_{k}^{2} \Rightarrow 1 \text { as } n \rightarrow \infty .
$$

Then

$$
\frac{S_{n}}{D_{n}} \Rightarrow N(0,1) \text { as } n \rightarrow \infty .
$$

Notice that the normalizer $D_{n}$ is rather complicated and contains the slowly varying function $H(x)$. By combining however the convergences in (15) and (16) it is easy to see that we can use in applications the selfnormalized form of this theorem.

$$
\frac{S_{n}}{\left(\sum_{k} b_{n k}^{2} \xi_{k}^{2}\right)^{1 / 2}} \Rightarrow N(0,1) \text { as } n \rightarrow \infty \text {. }
$$

By simple arguments $D_{n}$ in (16) can also be replaced by $\sqrt{\pi / 2} \mathbb{E}\left(\left|S_{n}\right|\right)$, or a consistent estimator of this quantity.

We mention now sufficient conditions for the validity of (15).

Theorem 2.4 Assume that $\left(\xi_{k}\right)_{k \in \mathbb{Z}}$ is a sequence of identically distributed martingale differences that satisfy (2), the coefficients $\left(a_{i}\right)$ are as in Theorem 2.3 and one of the conditions $\left(M_{1}\right)$ or $\left(M_{2}\right)$ of Proposition 2.1 is satisfied. Then both the central limit theorem (16) and its selfnormalized form (17) hold.

In the independent case, by combining theorem (2.4) with the result on selfnormalized CLT in Giné et al. (1997), we have: 
Theorem 2.5 Let $\left(\xi_{k}\right)_{k \in \mathbb{Z}}$ be a sequence of independent and identically distributed centered random variables. Then the following three statements are equivalent:

(1) $\xi_{0}$ satisfies condition (2).

(2) For any sequence of constants $\left(a_{n}\right)_{n \in \mathbb{Z}}$ satisfying (13) and $\sum_{k} b_{n k}^{2} \rightarrow \infty$ the CLT in (16) holds.

(3) For any sequence of constants $\left(a_{n}\right)_{n \in \mathbb{Z}}$ satisfying (13) and $\sum_{k} b_{n k}^{2} \rightarrow \infty$ the selfnormalized CLT in (17) holds.

This theorem is an extension of Theorem 18.6.5 in Ibragimov and Linnik (1971) from i.i.d. innovations with finite second moment to innovations in the domain of attraction of a normal law. It positively answers the question on the stability of the central limit theorem for i.i.d. variables under formation of linear sums. The implication $(2) \rightarrow(3)$ also follows by Mason (2005).

The casual linear process is obtained when $a_{i}=0$ for $i \leq 0$. Then $X_{0}=$ $\sum_{j=-\infty}^{0} a_{j} \xi_{j}$ is well defined if and only if

$$
\sum_{j \geq 0} a_{j}^{2} H\left(\left|a_{j}\right|^{-1}\right)<\infty
$$

For this case the coefficients $b_{n j}$ have the following expression

$$
\begin{aligned}
& b_{n j}=a_{1}+\ldots+a_{j} \text { for } j<n \\
& b_{n j}=a_{j-n+1}+\ldots+a_{j} \text { for } j \geq n
\end{aligned}
$$

and with this notation,

$$
S_{n}=\sum_{i=1}^{\infty} b_{n i} \xi_{n-i} .
$$

For particular casual linear processes with coefficients $a_{i}=i^{-\alpha} L(i)$, where $1 / 2<\alpha<1$ and $L(i)$ is a slowly varying function at $\infty$ in the strong sense (i.e. there is $h(t)$ continuous such that $L(n)=h(n)$ and $h(t)$ is slowly varying), the normalizer can be made more precise. Peligrad and Sang (2010) studied the case of i.i.d. innovations and showed that

$$
D_{n}^{2} \sim c_{\alpha} H\left(\eta_{n}\right) n^{3-2 \alpha} L^{2}(n)
$$

where $c_{\alpha}=(1-\alpha)^{-2} \int_{0}^{\infty}\left[x^{1-\alpha}-\max (x-1,0)^{1-\alpha}\right]^{2} d x$ and $\eta_{n}$ is defined by

$$
\eta_{j}=\inf \left\{s>1: \frac{H(s)}{s^{2}} \leq \frac{1}{j}\right\}, \quad j=1,2, \cdots
$$

Furthermore, in this context, Peligrad and Sang (2010) showed that the selfnormalizer can be estimated by observing only the variables $X_{k}$. More precisely they showed that $c_{\alpha} n^{2} a_{n}^{2} V_{n}^{2} /\left(A^{2} D_{n}^{2}\right) \Rightarrow 1$, where $V_{n}^{2}=\sum_{i=1}^{n} X_{i}^{2}$ and $A^{2}=\sum_{i=1}^{\infty} a_{i}^{2}$. Therefore

$$
\frac{S_{n}}{n a_{n} V_{n}} \Rightarrow N\left(0, \frac{c_{\alpha}}{A^{2}}\right)
$$


By combining this result with Theorem 2.3 we notice that for this case we have the following striking law of large numbers:

$$
\frac{A^{2} \sum_{i=1}^{\infty} b_{n i}^{2} \xi_{n-i}^{2}}{c_{\alpha} n^{2} a_{n}^{2} V_{n}^{2}} \Rightarrow 1 \text { as } n \rightarrow \infty .
$$

Another particular case of linear processes with i.i.d. innovations in the domain of attraction of a normal law was studied by Kulik (2006), under the condition $\sum_{j>0}\left|a_{j}\right|<\infty$. For this case his result is:

$$
\frac{S_{n}}{V_{n}} \Rightarrow N\left(0, \frac{\left|\sum_{i>0} a_{i}\right|^{2}}{A^{2}}\right) .
$$

It is an interesting related question to extend Kulik's result to martingale differences. We shall not pursue this path here where the goal is to consider general coefficients.

We shall also study the case of weak dependent random variables whose definition is based on the maximum coefficient of correlation.

Definition 2.1 Let $\mathcal{A}$ and $\mathcal{B}$ be two $\sigma$-algebras of events and define

$$
\rho(\mathcal{A}, \mathcal{B})=\sup _{f \in \mathbb{L}_{2}(\mathcal{A}), g \in \mathbb{L}_{2}(\mathcal{B})}|\operatorname{corr}(f, g)|
$$

where $\mathbb{L}_{2}(\mathcal{A})$ denotes the the class of random variables that are $\mathcal{A}$-measurable and square integrable.

Definition 2.2 Let $\left(\xi_{k}\right)_{k \in \mathbb{Z}}$ be a sequence of random variables and let $\mathcal{F}_{n}^{m}=$ $\sigma\left(\xi_{i}, n \leq i \leq m\right)$. We call the sequence $\rho$-mixing if

$$
\rho_{n}=\sup _{k} \rho\left(\mathcal{F}_{1}^{k}, \mathcal{F}_{k+n}^{\infty}\right) \rightarrow 0 \text { as } n \rightarrow \infty .
$$

This class is significant for studying functions of Gaussian processes, as well as additive functionals of Markov processes. A convenient reference for basic properties and the computation of these coefficients for functions of Markov chains and functions of Gaussian processes is Bradley (2007), chapters 7, 9 and 27.

The next theorem solves the same problem as Theorem 2.1 for this class of dependent random variables. The conditions imposed to the variables and mixing rates are similar to those used by Bradley (1988) who studied the central limit theorem for partial sums of stationary $\rho$-mixing sequences under (2). Bradley's result was extended in Shao (1993) in several directions, but still for partial sums. Our theorem extends Theorem 1 of Bradley (1988) from equal weights to linear processes and Theorem 2.2 (b) in Peligrad and Utev (1997) to variables with infinite second moment.

Theorem 2.6 Let $\left(\xi_{k}\right)$ be a sequence of centered identically distributed random variables satisfying (2). Assume that $\left(\xi_{k}\right)$ is $\rho$-mixing with $\sum_{k} \rho\left(2^{k}\right)<\infty$ and 
$\rho(1)<1$.

Let $\left(c_{n k}\right)$ be a triangular array of real numbers satisfying

$$
\sup _{n} \sum_{k=1}^{m_{n}} c_{n k}^{2} H\left(\left|c_{n k}\right|^{-1}\right)<\infty \text { and } \max _{1 \leq k \leq m_{n}} c_{n k}^{2} H\left(\left|c_{n k}\right|^{-1}\right) \rightarrow 0 \text { as } n \rightarrow \infty \text {. }
$$

Then

$$
\frac{1}{B_{n}} \sum_{k=1}^{m_{n}} c_{n k} \xi_{k} \Rightarrow N(0,1) \text { as } n \rightarrow \infty,
$$

where $B_{n}=(\pi / 2)^{1 / 2} \mathbb{E}\left|\sum_{k=1}^{m_{n}} c_{n k} \xi_{k}\right|$.

\section{Proofs}

\section{Proof of Theorem 2.1.}

The proof of Theorem 2.1 involves a few steps. Define

$$
\xi_{i}^{\prime}=\xi_{n i}^{\prime}=\xi_{i} I\left(\left|c_{n i} \xi_{i}\right| \leq 1\right)-\mathbb{E}_{i-1}\left(\xi_{i} I\left(\left|c_{n i} \xi_{i}\right| \leq 1\right)\right)
$$

and

$$
\xi_{i}^{\prime \prime}=\xi_{n i}^{\prime \prime}=\xi_{i} I\left(\left|c_{n i} \xi_{i}\right|>1\right)-\mathbb{E}_{i-1}\left(\xi_{i} I\left(\left|c_{n i} \xi_{i}\right|>1\right)\right),
$$

where we used the notation $\mathbb{E}_{i}(X)$ instead of $\mathbb{E}\left(X \mid \mathcal{F}_{i}\right)$.

We show now that

$$
\sum_{k=1}^{m_{n}} c_{n k} \xi_{k}^{\prime \prime} \Rightarrow 0
$$

This is true because by the item 2 of Lemma 4.1 and (4),

$$
\begin{gathered}
\mathbb{E}\left|\sum_{k=1}^{m_{n}} c_{n k} \xi_{k}^{\prime \prime}\right| \leq 2 \sum_{i=1}^{m_{n}}\left|c_{n i}\right| \mathbb{E}\left(\left|\xi_{0}\right| I\left(\left|c_{n i} \xi_{0}\right|>1\right)\right) \\
=o\left(\sum_{i=1}^{m_{n}} c_{n i}^{2} H\left(\left|c_{n i}\right|^{-1}\right)=o(1) \text { as } n \rightarrow \infty\right.
\end{gathered}
$$

To prove the theorem, by Theorem 3.1 in Billingsley (1999) and (21), it is enough to study the limiting distribution for the linear process associated to $\left(\xi_{i}^{\prime}\right)$.

We shall verify the sufficient conditions for the CLT for sums of a triangular array of martingale differences with finite second moment, given for convenience in Theorem 4.1 in the Appendix. We start by verifying the point $(a)$ of Theorem 4.1. Fix $0<\varepsilon<1$ and notice that by the properties of conditional expectations 
and item 4 in Lemma 4.1, we have:

$$
\begin{gathered}
\mathbb{E}\left(\max _{1 \leq i \leq m_{n}}\left|c_{n i} \xi_{i}^{\prime}\right|^{2}\right) \leq \varepsilon^{2}+\sum_{k=1}^{m_{n}} c_{n k}^{2} \mathbb{E}\left(\left(\xi_{k}^{\prime}\right)^{2} I\left(\left|c_{n k} \xi_{k}^{\prime}\right|>\varepsilon\right)\right) \\
\leq \varepsilon^{2}+\frac{1}{\varepsilon} \sum_{k=1}^{m_{n}}\left|c_{n k}\right|^{3} \mathbb{E}\left|\xi_{k}^{\prime}\right|^{3} \leq \varepsilon^{2}+\frac{8}{\varepsilon} \sum_{k=1}^{m_{n}}\left|c_{n k}\right|^{3} \mathbb{E}\left(\left|\xi_{k}\right|^{3} I\left(\left|c_{n k} \xi_{k}\right| \leq 1\right)\right) \\
\leq \varepsilon^{2}+\frac{8}{\varepsilon} o\left(\sum_{k=1}^{m_{n}}\left|c_{n k}\right|^{2} H\left(\left|c_{n k}\right|^{-1}\right) \text { as } n \rightarrow \infty .\right.
\end{gathered}
$$

Now we take into account condition (4) and obtain $\mathbb{E}\left(\max _{1 \leq i \leq m_{n}}\left|c_{n i} \xi_{i}^{\prime}\right|^{2}\right) \rightarrow 0$ by letting first $n \rightarrow \infty$ followed by $\varepsilon \rightarrow 0$.

In order to verify the item $(b)$ of Theorem 4.1, we have to study the limit in probability of $\sum_{k=1}^{m_{n}} c_{n k}^{2}\left(\xi_{k}^{\prime}\right)^{2}$. We start from the decomposition

$$
\begin{aligned}
& \sum_{k=1}^{m_{n}} c_{n k}^{2}\left(\xi_{k}^{\prime}\right)^{2}=\sum_{k=1}^{m_{n}} c_{n k}^{2} \xi_{k}^{2} I\left(\left|c_{n k} \xi_{k}\right| \leq 1\right)+\sum_{i=1}^{m_{n}} c_{n i}^{2} \mathbb{E}_{i-1}^{2}\left(\xi_{i} I\left(\left|c_{n i} \xi_{i}\right| \leq 1\right)\right) \\
& \quad+2 \sum_{k=1}^{m_{n}} c_{n k}^{2} \xi_{k} I\left(\left|c_{n k} \xi_{k}\right| \leq 1\right) \mathbb{E}_{k-1}\left(\xi_{k} I\left(\left|c_{n k} \xi_{k}\right|>1\right)\right)=A+B+2 C .
\end{aligned}
$$

We shall show that it is enough to analyze the first term by the following simple argument.

We discuss now the term $B$. By the fact that $c_{n i}^{2}\left(\mathbb{E}_{i-1}\left(\xi_{i} I\left(\left|c_{n i} \xi_{i}\right| \leq 1\right)\right)\right)^{2} \leq$ 1 a.s. we obtain

$$
B \leq \sum_{i=1}^{m_{n}}\left|c_{n i} \mathbb{E}_{i-1}\left(\xi_{i} I\left(\left|c_{n i} \xi_{i}\right| \leq 1\right)\right)\right| \text { a.s. }
$$

By the martingale property $\mathbb{E}_{i-1}\left(\xi_{i} I\left(\left|c_{n i} \xi_{i}\right| \leq 1\right)\right)=\mathbb{E}_{i-1}\left(\xi_{i} I\left(\left|c_{n i} \xi_{i}\right|>1\right)\right)$ a.s. and taking into account (21) and the properties of conditional expectation,

$$
\begin{aligned}
\mathbb{E}(B) & \leq \mathbb{E}\left(\sum_{i=1}^{m_{n}}\left|c_{n i} \mathbb{E}_{i-1}\left(\xi_{i} I\left(\left|c_{n i} \xi_{i}\right|>1\right)\right)\right|\right) \\
& \leq \sum_{i=1}^{m_{n}}\left|c_{n i}\right| \mathbb{E}\left(\left|\xi_{0}\right| I\left(\left|c_{n i} \xi_{0}\right|>1\right)\right)=o(1) \text { as } n \rightarrow \infty .
\end{aligned}
$$

Then, by Cauchy Schwarz inequality, condition (4) and (22), we get

$$
\mathbb{E}(C) \leq(\mathbb{E}(A) \mathbb{E}(B))^{1 / 2} \rightarrow 0 \text { as } n \rightarrow \infty .
$$

By these arguments, the limit in probability of $\sum_{k=1}^{m_{n}} c_{n k}^{2}\left(\xi_{k}^{\prime}\right)^{2}$ coincides to the limit of $\sum_{k=1}^{m_{n}} c_{n k}^{2} \xi_{k}^{2} I\left(\left|c_{n k} \xi_{k}\right| \leq 1\right)$. Notice now that

$$
\sum_{k=1}^{m_{n}} c_{n k}^{2} \xi_{k}^{2} I\left(\left|c_{n k} \xi_{k}\right| \leq 1\right)-1=\left(\sum_{k=1}^{m_{n}} c_{n k}^{2} \xi_{k}^{2}-1\right)+\sum_{k=1}^{m_{n}} c_{n k}^{2} \xi_{k}^{2} I\left(\left|c_{n k} \xi_{k}\right|>1\right) .
$$


By the martingale inequality in Burkholder (1966) we obtain that there is a positive constant $c$, such that for any $\varepsilon>0$

$$
\mathbb{P}\left(\sum_{k=1}^{m_{n}} c_{n k}^{2}\left(\xi_{k}^{\prime \prime}\right)^{2}>\varepsilon / 2\right) \leq \frac{c}{\varepsilon^{2}} \mathbb{E}\left|\sum_{k=1}^{m_{n}} c_{n k}\left(\xi_{k}^{\prime \prime}\right)\right|
$$

which, combined with (21) and the arguments in (22) gives

$$
\sum_{k=1}^{m_{n}} c_{n k}^{2} \xi_{k}^{2} I\left(\left|c_{n k} \xi_{k}\right|>1\right) \Rightarrow 0 .
$$

We just have to take into account condition (5) to conclude

$$
\sum_{k=1}^{m_{n}} c_{n k}^{2} \xi_{k}^{2} I\left(\left|c_{n k} \xi_{k}\right| \leq 1\right) \Rightarrow 1
$$

and then, by the above considerations, $\sum_{k=1}^{m_{n}} c_{n k}^{2}\left(\xi_{k}^{\prime}\right)^{2} \Rightarrow 1$.

The conclusion of this theorem follows by using the Theorem $4.1 \diamond$

\section{Proof of Proposition 2.1.}

We assume that $\left(\mathrm{M}_{1}\right)$ holds. Because of (23) it is enough to prove

$$
\sum_{k=1}^{m_{n}} c_{n k}^{2} \xi_{k}^{2} I\left(\left|c_{n k} \xi_{k}\right| \leq 1\right) \Rightarrow 1
$$

We start by computing the variance of $\sum_{k=1}^{m_{n}} c_{n k}^{2} \xi_{k}^{2} I\left(\left|c_{n k} \xi_{k}\right| \leq 1\right)$ and, taking into account the variables are identically distributed, we majorate the covariances by using the coefficients $\psi_{i}$ defined in Condition $\left(\mathrm{M}_{1}\right)$.

$$
\begin{gathered}
\operatorname{var}\left(\sum_{k=1}^{m_{n}} c_{n k}^{2} \xi_{k}^{2} I\left(\left|c_{n k} \xi_{k}\right| \leq 1\right)\right) \leq \sum_{k=1}^{m_{n}} c_{n k}^{4} \mathbb{E}\left(\xi_{0}^{4} I\left(\left|c_{n k} \xi_{0}\right| \leq 1\right)\right) \\
+2 \sum_{i=1}^{m_{n}-1} \psi_{i} \sum_{k=1}^{m_{n}-i} c_{n k}^{2} c_{n, k+i}^{2} \mathbb{E}\left(\xi_{0}^{2} I\left(\left|c_{n k} \xi_{0}\right| \leq 1\right)\right) \mathbb{E}\left(\xi_{0}^{2} I\left(\left|c_{n, k+i} \xi_{0}\right| \leq 1\right)\right)=D+2 E .
\end{gathered}
$$

By the item 4 of Lemma 4.1 .

$$
D=\sum_{k=1}^{m_{n}} c_{n k}^{4} \mathbb{E}\left(\xi_{0}^{4} I\left(\left|c_{n k} \xi_{0}\right| \leq 1\right)\right)=o\left(\sum_{k=1}^{m_{n}} c_{n k}^{2} H\left(\left|c_{n k}\right|^{-1}\right)\right)=o(1) \text { as } n \rightarrow \infty .
$$

In order to estimate the second term we split the sum in two, one up to $h$ and 
another after $h$, where $h$ is an integer.

$$
\begin{aligned}
E & =\sum_{i=1}^{m_{n}-1} \psi_{i} \sum_{k=1}^{m_{n}-i} c_{n k}^{2} c_{n, k+i}^{2} H\left(\left|c_{n k}\right|^{-1}\right) H\left(\left|c_{n, k+i}\right|^{-1}\right) \leq \\
& h \max _{1 \leq i \leq h} \sum_{k=1}^{m_{n}-i} c_{n k}^{2} c_{n, k+i}^{2} H\left(\left|c_{n k}\right|^{-1}\right) H\left(\left|c_{n k+i}\right|^{-1}\right) \\
& +\max _{h \leq i \leq m_{n}} \psi_{i} \sum_{i=1}^{m_{n}-1} \sum_{k=1}^{m_{n}-i} c_{n k}^{2} c_{n, k+i}^{2} H\left(\left|c_{n k}\right|^{-1}\right) H\left(\left|c_{n, k+i}\right|^{-1}\right) \\
& \leq h \max _{1 \leq k \leq m_{n}} c_{n k}^{2} H\left(\left|c_{n k}\right|^{-1}\right)+\max _{h \leq i \leq m_{n}} \psi_{i} .
\end{aligned}
$$

By letting $n \rightarrow \infty$ and then $h \rightarrow \infty$, by taking into account conditions (44) and $\left(\mathrm{M}_{1}\right)$, we obtain $E \rightarrow 0$ as $n \rightarrow \infty$. Then, clearly by the above considerations $\operatorname{var}\left(\sum_{k=1}^{m_{n}} c_{n k}^{2}\left(\xi_{k}^{\prime}\right)^{2}\right) \rightarrow 0$ that further implies by condition (4) that (5) holds under $\left(\mathrm{M}_{1}\right)$.

The proof of this proposition under $\left(\mathrm{M}_{2}\right)$ is similar. Because $c_{n i}^{2} \mathbb{E}\left(\xi_{i}^{2} I\left(\left|c_{n i} \xi_{i}\right| \leq\right.\right.$ 1)) $\leq 1$,

$$
\begin{gathered}
\operatorname{var}\left(\sum_{k=1}^{m_{n}} c_{n k}^{2} \xi_{k}^{2} I\left(\left|c_{n k_{k}} \xi\right| \leq 1\right) \leq \sum_{k=1}^{m_{n}} c_{n k}^{4} \mathbb{E}\left(\xi_{0}^{4} I\left(\left|c_{n k} \xi_{0}\right| \leq 1\right)\right)\right. \\
\quad+2 \sum_{i=1}^{m_{n}-1} \sum_{k=i+1}^{m_{n}} \varphi_{k-i} c_{n k}^{2} \mathbb{E}\left(\xi_{0}^{2} I\left(\left|c_{n k} \xi_{0}\right| \leq 1\right)\right)
\end{gathered}
$$

The first term in the right hand side is treated as before. For $h$ fixed we easily obtain

$$
\begin{aligned}
& \sum_{i=1}^{m_{n}-1} \sum_{k=i+1}^{m_{n}} \varphi_{k-i} c_{n k}^{2} \mathbb{E} \xi_{0}^{2} I\left(\left|c_{n k} \xi_{0}\right| \leq 1\right) \leq h \max _{1 \leq k \leq m_{n}} c_{n k}^{2} H\left(\left|c_{n k}\right|^{-1}\right) \sum_{k=1}^{m_{n}} \varphi_{k} \\
& \quad+\sum_{i=h}^{\infty} \varphi_{i} \sum_{k=1}^{m_{n}} c_{n k}^{2} \mathbb{E} \xi_{0}^{2} I\left(\left|c_{n k} \xi_{0}\right| \leq 1\right)
\end{aligned}
$$

and the result follows by (44) and condition $\left(\mathrm{M}_{2}\right)$ by letting first $n \rightarrow \infty$ followed by $h \rightarrow \infty$. $\diamond$

\section{Proof of Proposition 2.2}

By the three series theorem for martingales, (Theorem 2.16 in Hall and Heyde, 1980) $X_{0}$ exists in the almost sure sense if and only if:

1. $\sum_{i} \mathbb{P}\left(\left|a_{i} \xi_{i}\right|>1 \mid \mathcal{F}_{i-1}\right)<\infty$ a.s.,

2. $\sum_{i} \mathbb{E}\left(a_{i} \xi_{i} I\left(\left|a_{i} \xi_{i}\right| \leq 1\right) \mid \mathcal{F}_{i-1}\right)$ converges a.s., 


\section{3. $\sum_{i} \operatorname{Var}\left(a_{i} \xi_{i} I\left(\left|a_{i} \xi_{i}\right| \leq 1 \mid \mathcal{F}_{i-1}\right)\right)<\infty$ a.s.}

Notice that, by taking into account Convention 1, the fact that the variables are identically distributed and item 2 in Lemma 4.1 from the appendix,

$$
\sum_{i} \mathbb{P}\left(\left|a_{i} \xi_{i}\right|>1\right)=\sum_{i} \mathbb{P}\left(\left|a_{i} \xi_{0}\right|>1\right)=\sum_{i} a_{i}^{2} o\left(H\left(\left|a_{i}\right|^{-1}\right)\right)<\infty
$$

and this easily implies 1 .

Then by item 3 of Lemma 4.1 and again by the fact that the variables are identically distributed,

$\mid \sum_{i} \mathbb{E}\left(a_{i} \xi_{i} I\left(\left|a_{i} \xi_{i}\right| \leq 1\right)\left|\leq \sum_{i}\right| a_{i} \mid \mathbb{E}\left(\left|\xi_{0}\right| I\left(\left|a_{i} \xi_{0}\right|>1\right)\right)=\sum_{i} a_{i}^{2} o\left(H\left(\left|a_{i}\right|^{-1}\right)<\infty\right.\right.$.

This implies

$$
\sum_{i} \mathbb{E}\left(\left|a_{i} \xi_{i}\right| I\left(\left|a_{i} \xi_{i}\right| \leq 1\right) \mid \mathcal{F}_{i-1}\right)<\infty \text { a.s. }
$$

and 2. follows.

Finally,

$$
\sum_{i} \mathbb{E}\left(a_{i}^{2} \xi_{i}^{2} I\left(\left|a_{i} \xi_{i}\right| \leq 1\right)\right)=\sum_{i} a_{i}^{2} \mathbb{E}\left(\xi_{0}^{2} I\left(\left|a_{i} \xi_{0}\right| \leq 1\right)\right)=\sum_{i} a_{i}^{2} H\left(\left|a_{i}\right|^{-1}\right) .
$$

Then

$$
\sum_{i} a_{i}^{2} \mathbb{E}\left(\xi_{i}^{2} I\left(\left|a_{i} \xi_{i}\right| \leq 1\right) \mid \mathcal{F}_{i-1}\right)<\infty \text { a.s. }
$$

and together with (24) gives 3. For the i.i.d. case the proof is similar and it is based on the i.i.d. version of the three series theorem. $\diamond$

\section{Proof of Theorem 2.3}

We start by rewriting $S_{n}$ as in relation (14) by changing the order of summation.

$$
S_{n}=\sum_{k=1}^{n} X_{k}=\sum_{j=-\infty}^{\infty}\left(\sum_{k=1}^{n} a_{k+j}\right) \xi_{j}=\sum_{j=-\infty}^{\infty} b_{n j} \xi_{j} .
$$

We shall verify the conditions of Theorem 2.1.

According to Corollary (2.1) it is sufficient to show that

$$
\sup _{k} \frac{b_{n k}^{2}}{D_{n}^{2}} H\left(\frac{D_{n}}{\left|b_{n k}\right|}\right) \rightarrow 0 \text { as } n \rightarrow \infty
$$

where

$$
D_{n}=\inf \left\{x \geq 1: \sum_{k} \frac{b_{n k}^{2}}{x^{2}} H\left(\frac{x}{\left|b_{n k}\right|}\right) \leq 1\right\}
$$


Notice that condition (13) implies $\sum_{k} a_{k}^{2}<\infty$. Therefore we can apply the argument from Peligrad and Utev (1997, pages 448-449) and obtain

$$
\sup _{k} \frac{b_{n k}^{2}}{\sum_{i} b_{n i}^{2}} \rightarrow 0 \text { as } n \rightarrow \infty
$$

since we imposed $\sum_{k} b_{n k}^{2} \rightarrow \infty$. Notice that by taking into account Convention 2 we obviously have

$$
\frac{1}{\sum_{k} b_{n k}^{2}} \sum_{k} b_{n k}^{2} H\left(\frac{\left(\sum_{k=1} b_{n k}^{2}\right)^{1 / 2}}{\left|b_{n k}\right|}\right) \geq 1 .
$$

By the definition of $D_{n}$, this implies

$$
D_{n}^{2} \geq \sum_{k} b_{n k}^{2}
$$

whence, by (25)

$$
\sup _{k} \frac{b_{n k}^{2}}{D_{n}^{2}} \rightarrow 0 \text { as } n \rightarrow \infty
$$

Now, by the properties of slowly varying functions, for $\varepsilon>0$, we know that $H(x)=o\left(x^{\varepsilon}\right)$ as $x \rightarrow \infty$. We then obtain

$$
\sup _{k} \frac{b_{n k}^{2}}{D_{n}^{2}} H\left(\frac{D_{n}}{\left|b_{n k}\right|}\right)=o(1) \text { as } n \rightarrow \infty .
$$

This completes the proof of this Theorem. $\diamond$

\section{Proof of Theorem 2.6}

In order to prove Theorem 2.6 we start by the truncation argument

$$
\xi_{n i}^{\prime}=\xi_{i} I\left(\left|c_{n i} \xi_{i}\right| \leq 1\right)-\mathbb{E}\left(\xi_{i} I\left(\left|c_{n i} \xi_{i}\right| \leq 1\right)\right)
$$

and

$$
\xi_{n i}^{\prime \prime}=\xi_{i} I\left(\left|c_{n i} \xi_{i}\right|>1\right)-\mathbb{E}\left(\xi_{i} I\left(\left|c_{n i} \xi_{i}\right|>1\right)\right) .
$$

First, by relation (21), we have

$$
E\left|\sum_{k=1}^{m_{n}} c_{n k} \xi_{k}^{\prime \prime}\right| \rightarrow 0
$$

whence, by Theorem 3.1 in Billingsley (1999), the proof is reduced to studying the asymptotic behavior of $\sum_{k=1}^{m_{n}} c_{n k} \xi_{k}^{\prime}$.

According to Theorem 4.1 and Theorem 5.5 in Utev (1990), given for convenience in the appendix (Theorem 4.2), we have only to verify the Lindeberg's condition. Denote $\left(\sigma_{n}^{\prime}\right)^{2}=\mathbb{E}\left(\sum_{k=1}^{m_{n}} c_{n k} \xi_{k}^{\prime}\right)^{2}$. Our conditions on the mixing coefficients allows us by relation (29) to bound $\left(\sigma_{n}^{\prime}\right)^{2}$ above and below by the sum 
of squares of the variance of individual summands; so we can find two positive constants $C_{1}<C_{2}$ such that

$$
C_{1} \sum_{i=1}^{m_{n}} c_{n i}^{2} H\left(\left|c_{n i}\right|^{-1}\right) \leq\left(\sigma_{n}^{\prime}\right)^{2} \leq C_{2} \sum_{i=1}^{m_{n}} c_{n i}^{2} H\left(\left|c_{n i}\right|^{-1}\right) .
$$

Lindeberg's condition is satisfied because Lyapunov's condition is satisfied. To see this, by (27) we have

$$
\frac{1}{\left(\sigma_{n}^{\prime}\right)^{2}} \sum_{i=1}^{m_{n}} c_{n i}^{3} \mathbb{E}\left(\left|\xi_{0}\right|^{3} I\left(\left|c_{n i} \xi\right| \leq 1\right)\right) \leq \frac{1}{\left(\sigma_{n}^{\prime}\right)^{2}} \sum_{i=1}^{m_{n}} c_{n i}^{2} o\left(H\left(\left|c_{n i}\right|^{-1}\right)\right)=o(1) .
$$

The central limit theorem follows and we have

$$
\left(\sum_{k=1}^{m_{n}} c_{n k} \xi_{k}^{\prime}\right) / \sigma_{n}^{\prime} \Rightarrow N(0,1) \text { as } n \rightarrow \infty .
$$

Now, $\left(\sum_{k=1}^{m_{n}} c_{n k} \xi_{k}^{\prime}\right) / \sigma_{n}^{\prime}$ is uniformly integrable and by Theorem 3.4 in Billingsley (1999), this implies that $\mathbb{E}\left(\left|\sum_{k=1}^{m_{n}} c_{n k} \xi_{k}^{\prime}\right| / \sigma_{n}^{\prime}\right) \rightarrow \sqrt{2 / \pi}$. By taking now into account (26) we obtain $\mathbb{E}\left(\left|\sum_{k=1}^{m_{n}} c_{n k} \xi_{k}\right| / \sigma_{n}^{\prime}\right) \rightarrow \sqrt{2 / \pi}$, that combined with (28) and (26), leads to the conclusion of this theorem. $\diamond$

\section{Appendix}

We mention first a lemma which contains some equivalent formulation for variables in the domains of attraction of normal law. It is Lemma 1 in Csörgő et al (2003).

Lemma 4.1 Let $H(x):=\mathbb{E}\left(X^{2} I(|X| \leq x)\right)$. The following statements are equivalent.

1. $H(x)$ is a slowly varying function at $\infty$;

2. $\mathbb{P}(|X|>x)=o\left(x^{-2} H(x)\right)$;

3. $\mathbb{E}(|X| I(|X|>x))=o\left(x^{-1} H(x)\right)$;

4. $\mathbb{E}\left(|X|^{\alpha} I(|X| \leq x)\right)=o\left(x^{\alpha-2} H(x)\right)$ for $\alpha>2$.

The following theorem is a simplified version of Theorem 3.2 from Hall and Heyde (1980). See also Relation 2.18 and (M) in Gaenssler and Haeusler (1986).

Theorem 4.1 Let $\left(D_{n i}\right)_{1 \leq i \leq k_{n}}$ be a square integrable martingale difference and $\left(\mathcal{F}_{i}\right)$ a filtration of sigma algebras such that for each, $n$ and $1 \leq i \leq k_{n}, D_{n i}$ is $\mathcal{F}_{i}$ measurable. Suppose that

(a) $\max _{1 \leq i \leq k_{n}}\left|D_{n i}\right|$ is uniformly integrable;

(b) $\sum_{i=1}^{k_{n}} D_{n i}^{2} \Rightarrow 1$ as $n \rightarrow \infty$;

Then $S_{n} \Rightarrow N(0,1)$ as $n \rightarrow \infty$ where $S_{n}=\sum_{i=1}^{k_{n}} X_{n i}$. 
By combining Theorem 4.1 and Theorem 5.5 in Utev (1990) we formulate a general result for triangular arrays of $\rho$-mixing random variables.

Theorem 4.2 Assume $\left(Y_{n k}\right)_{1 \leq k \leq m_{n}}$ is a triangular array of centered random variables with finite second moment such that $\rho(1)<1$ and $\sum_{k} \rho\left(2^{k}\right)<\infty$ where

$$
\rho(k)=\sup _{s \geq 1, n \geq 1} \rho\left(\sigma\left(Y_{n i} ; i \leq s\right), \sigma\left(Y_{n i} ; i \geq s+k\right)\right.
$$

Set $S_{n}=\sum_{i=1}^{m_{n}} Y_{n i}$ and denote $\operatorname{var}\left(S_{n}\right)=\sigma_{n}^{2}$. Then, there are two positive constants $C_{1}$ and $C_{2}$ such that

$$
C_{1} \sum_{i=1}^{m_{n}} \mathbb{E}\left(Y_{n i}^{2}\right) \leq \sigma_{n}^{2} \leq C_{2} \sum_{i=1}^{m_{n}} \mathbb{E}\left(Y_{n i}^{2}\right)
$$

Moreover if Lindeberg's condition is satisfied:

$$
\frac{1}{\sigma_{n}^{2}} \sum_{i=1}^{m_{n}} \mathbb{E}\left(Y_{n i}^{2} I\left(\left|Y_{n i}\right|>\varepsilon \sigma_{n}\right)\right) \rightarrow 0
$$

then

$$
\frac{1}{\sigma_{n}} \sum_{i=1}^{m_{n}} Y_{n i} \Rightarrow N(0,1) .
$$

\section{Acknowledgement}

The authors are grateful to the referees for their useful suggestions which improved the presentation of this paper.

\section{References}

[1] Araujo, A. and Giné, E. (1980). The Central Limit Theorem for Real and Banach Valued Random Variables. Wiley Series in Probability and Mathematical Statistics. John Wiley \& Sons, New York-Chichester-Brisbane.

[2] Beran, J. (1994). Statistics for long-memory processes. Monographs on Statistics and Applied Probability, 61. Chapman and Hall, New York.

[3] Billingsley, P. (1999). Convergence of Probability measures. Second edition, Wiley, New York.

[4] Bradley, R. C. (1988). A Central Limit Theorem for Stationary $\rho$-Mixing Sequences with Infinite Variance. Ann. Probab. 16 313-332.

[5] Bradley, R. C. (2007). Introduction to strong mixing conditions. Volumes 1-3, Kendrick Press. 
[6] Burkholder, D. L. (1966). Martingale transforms. Ann. Math. Statist. 37 1494-1504.

[7] Csörgö, M., Szyszkowicz, B. and Wang, Q. (2003). Donsker's theorem for self-normalized partial sums processes, Ann. Probab. 31 1228-1240.

[8] Feller, W. (1966). An Introduction to Probability Theory and Its Applications Vol. 2, Willey, New York.

[9] Gaenssler, P. and Haeusler, E. (1986). On martingale central limit theory. Dependence in Probability and Statistics, Birkhäuser, Boston, 303-334.

[10] Giné, E., Götze, F. and Mason, D. M. (1997). When is the Student tstatistic asymptotically standard normal? Ann. Probab. 25, 1514-1531.

[11] Gordin, M. I. (1969). The central limit theorem for stationary processes, Soviet. Math. Dokl. 10, 1174-1176.

[12] Hall P. and Heyde, C. C. (1980). Martingale Limit Theory and Its Applications, Academic Press.

[13] Jara, M., Komorowski, T. and Olla, S. (2009). Limit theorems for additive functionals of a Markov Chain. Ann. Appl. Probab. 2009, 19, 2270-2300

[14] Knight, K. (1991). Limit theory for M-estimates in an integrated infinite variance process. Econometric Theory 7, 200-212.

[15] Kulik, R. (2006). Limit theorems for self-normalized linear processes. Statistics and Probability Letters 76, 1947-1953.

[16] Ibragimov, I. A. and Linnik, Yu. V. (1971). Independent and Stationary Sequences of Random Variables, Wolters, Groningen.

[17] Mason, D.M. (2005). The asymptotic distribution of self-normalized triangular arrays. J. Theor. Probab. 18, 853-870.

[18] Mikosch, T. Gadrich, T. Kliippelberg C. and Adler, R. J. (1995). Parameter estimation for ARMA models with infinite variance innovations. Annals of Statistics 23, 305-326.

[19] Peligrad, M. and Utev, S. (1997). Central limit theorem for linear processes. Ann. Probab. 25, 443-456.

[20] Peligrad, M. and Utev, S. (2006). Central limit theorem for stationary linear processes. Ann. Probab. 34, 1608-1622.

[21] Peligrad, M. and Sang, H. (2010). Asymptotic Properties of SelfNormalized Linear Processes with Long Memory. arXiv:1006.1572

[22] Robinson, P. M. (1997). Large-sample inference for non parametric regression with dependent errors. Ann. Statist. 25, 2054-2083. 
[23] Shao, Q. (1993). On the invariance principle for $\rho$-mixing sequence of random variables with infinite variance. Chinese Ann. Math. Ser. B. 14, 27-42.

[24] Tyran-Kamińska, M. (2010). Functional limit theorems for linear processes in the domain of attraction of stable laws . Statistics and Probability Letters 77, 1535-1541.

[25] Utev, S. A. (1990). Central limit theorem for dependent random variables. Prob. Theory and Math. Stat. Vol. 2, B. Grigelionis et al. (eds.), VSP/Mokslas. 519-528.

[26] Volný, D (1993). Approximating martingales and central limit theorem for strictly stationary processe. Stoch. Proc. Appl. 44, 41-74.

[27] Wu, W. B. (2003). Additive functionals of infinite-variance moving averages. Statistica Sinica 13, 1259-1267. 